\title{
COMPARACIÓN DE LAS ESTRATEGIAS ANALGÉSICAS MÁS UTILIZADAS EN EL MANEJO DEL DOLOR POSTOPERATORIO EN EL HOSPITAL CLÍNICA BÍBLICA
}

Chaverri, Fernández, José Miguel ${ }^{1}$; Zavaleta Monestel, Esteban²; Díaz Madriz, José Pablo ${ }^{3}$; Ortiz Ureña Angie ${ }^{4}$; Álvarez Pacheco, Helga $^{5}$ y Zúñiga Morales, Hazel ${ }^{5}$

\begin{abstract}
1Departamento de Farmacología, Toxicología y Farmacodependencia de la Facultad de Farmacia de la Universidad de Costa Rica, Farmacéutico Clínico Hospital Clínica Bíblica, San José, Costa Rica

2Director de la Farmacia Hospital Clínica Bíblica, San José, Costa Rica

3Farmacéutico Clínico. Hospital Clínica Bíblica, San José, Costa Rica

4Departamento de Farmacología, Toxicología y Farmacodependencia de la Facultad de Farmacia de la Universidad de Costa Rica

5Estudiantes de Internado. Universidad de Ciencias Médicas UCIMED-Hospital Clínica Bíblica.
\end{abstract}

\begin{abstract}
Resumen:
Objetivo: Analizar la efectividad de las estrategias analgésicas para el manejo del dolor postoperatorio usadas en el Hospital Clínica Bíblica mediante la aplicación de la escala visual análoga (EVA) 24 horas después de la cirugía y en el período postoperatorio. Resultados: Se halló que las combinaciones más usadas en el período postoperatorio fueron 1. Dexketoprofeno + Metamizol, 2. Dexketoprofeno + Metamizol + Morfina y 3. Dexketoprofeno solo, en orden descendente; en relación con los valores en la EVA reportados existen diferencias estadísticamente significativas para las combinaciones 1 y 2 y 2 y 3 , ambos casos en detrimento de la combinación 2. Además se hallaron correlaciones con los valores en la EVA reportados de tipo positiva para la variable días de internamiento y de tipo negativa para la variable edad, así como valores menores en la EVA en pacientes masculinos y aquellos que recibieron premedicación. Conclusiones: Las estrategias para el manejo del dolor postoperatorio usadas el Hospital Clínica Bíblica son efectivas, con mejores resultados para combinaciones de dos analgésicos y la ausencia de combinaciones con opioides. Además, se debe considerar la afectación de los valores de la EVA en función de factores como la edad, género, días de estancia hospitalaria y aplicación de premedicación.
\end{abstract}

Palabras clave: escala visual analógica, dolor, postoperatorio, efectividad Recibido: 7 Enero 2015. Aceptado: 20 marzo 2015. Publicado: 15 Abril 2015.

Revista electrónica publicada por el Departamento de Farmacología de la Escuela de Medicina de la Universidad de Costa Rica, 2060 San José, Costa Rica. ${ }^{\circledR}$ All rights reserved. Licensed under a Creative Commons Unported License. 


\title{
COMPARISON OF THE MOST USED ANALGESIC STRATEGIES FOR POST-SURGICAL PAIN MANAGEMENT IN HOSPITAL CLÍNICA BÍBLICA
}

\begin{abstract}
:
Objective: Analyze the effectiveness of analgesic strategies used for post-surgical pain management in Hospital Clínica Bíblica for patients who reported perceived pain by answering the visual analogue pain scale (VAS) 24 hours after surgery and post-surgery. Results: The most used combinations were, in descending order: 1. Dexketoprofen+Metamizole, 2. Dexketoprofen+Metamizole+Morphine and 3. Only Dexketoprofen; there are statistically significant differences for combinations 1 and 2, and 2 and 3, on the downside for number 2. Correlations were found for VAS values, they were positive with days of hospitalization, negative with age, and lower VAS values in males and those who received medication prior to surgery. Conclusions: Pain management strategies used at Hospital Clínica Bíblica are effective, especially the combinations of two analgesics and the absence of combinations with opioids. The effect of age, sex, days of hospitalization and medication prior to surgery on values of perceived pain measured with VAS should be considered in postsurgery pain management strategies.
\end{abstract}

Key words: visual analogue pain scale, pain, post-surgical, effectiveness

\section{INTRODUCCIÓN}

Según la International Association of Study of Pain (IASP) "el dolor es una experiencia no placentera, sensorial y afectiva, asociada a daño tisular actual o potencial" [1]. Por su parte, los estándares de la Joint Commission International Accreditation Standards for Hospitals indican que el dolor debe ser tomado como el quinto signo vital necesario para establecer el diagnóstico del paciente, además de ser uno de los motivos de consulta más frecuente en la práctica clínica. Existen tres tipos de dolor; el dolor nociceptivo (somático y visceral), no nociceptivo (neuropático) y el dolor idiopático [1-4].

Por tanto, para clasificar o categorizar al dolor es necesario valorar su intensidad, localización y duración, para lo cual existen diferentes instrumentos, tales como escala visual análoga del dolor (conocida como EVA), que permite aproximar la intensidad del dolor descrito por el paciente con una buena reproducibilidad interobservadores. Esta escala consiste en una línea horizontal de 10 centímetros, en cuyos bordes se encuentran las expresiones extremas de un síntoma. En el extremo izquierdo se ubica la ausencia de este o la percepción del mismo en una menor intensidad y en el derecho la percepción con mayor intensidad. Al aplicarlo, se pide al paciente que marque dentro de la escala la ubicación que indique la intensidad del síntoma que está percibiendo y luego se mide con una regla graduada esta ubicación [3,5-7]; la localización proporciona una guía para la evaluación actual del problema de salud, la escogencia del tratamiento y la efectividad de la terapia seleccionada. El valor obtenido permite aproximar el tipo de dolor que presenta el paciente: un valor inferior a 4 indica un dolor leve o leve-moderado, un valor entre 4 y 6 implica la presencia de dolor moderado-grave, y

Revista electrónica publicada por el Departamento de Farmacología de la Escuela de Medicina de la Universidad de Costa Rica, 2060 San José, Costa Rica. ${ }^{\circledR}$ All rights reserved. Licensed under a Creative Commons Unported License. 
un valor superior a 6 implica la presencia de un dolor intenso $[3,5,6]$.

El manejo del dolor postoperatorio ha tenido avances significativos en años recientes; específicamente en la década de 1990 hubo un incremento en la concientización acerca de la importancia de mejorar las estrategias analgésicas utilizadas, para brindar a los pacientes un mayor control del dolor y una mejor calidad en la atención recibida $[3,8]$.

Así, existen varios factores a considerar para la selección de una adecuada analgesia; por ejemplo la ruta de administración (se espera que el bolo intravenoso sea la vía utilizada con mayor frecuencia en el postoperatorio), la frecuencia de la dosis, el manejo y la tolerancia a los posibles efectos adversos, la variación individual del paciente, la edad, género, grupo étnico $y$ experiencia previa de uso del analgésico [5,8].

Como consecuencia del deficiente manejo del dolor del paciente oncológico, en la década de 1990 la Organización Mundial de la Salud (OMS) promovió el uso de la escala analgésica y a partir de las recomendaciones emanadas se establecieron las estrategias mono y multimodales (terapias combinadas) que pretenden conseguir un adecuado abordaje del según el dolor referido o a percibir, tal como se detalla en la figura $1[2,5,8,9]$.

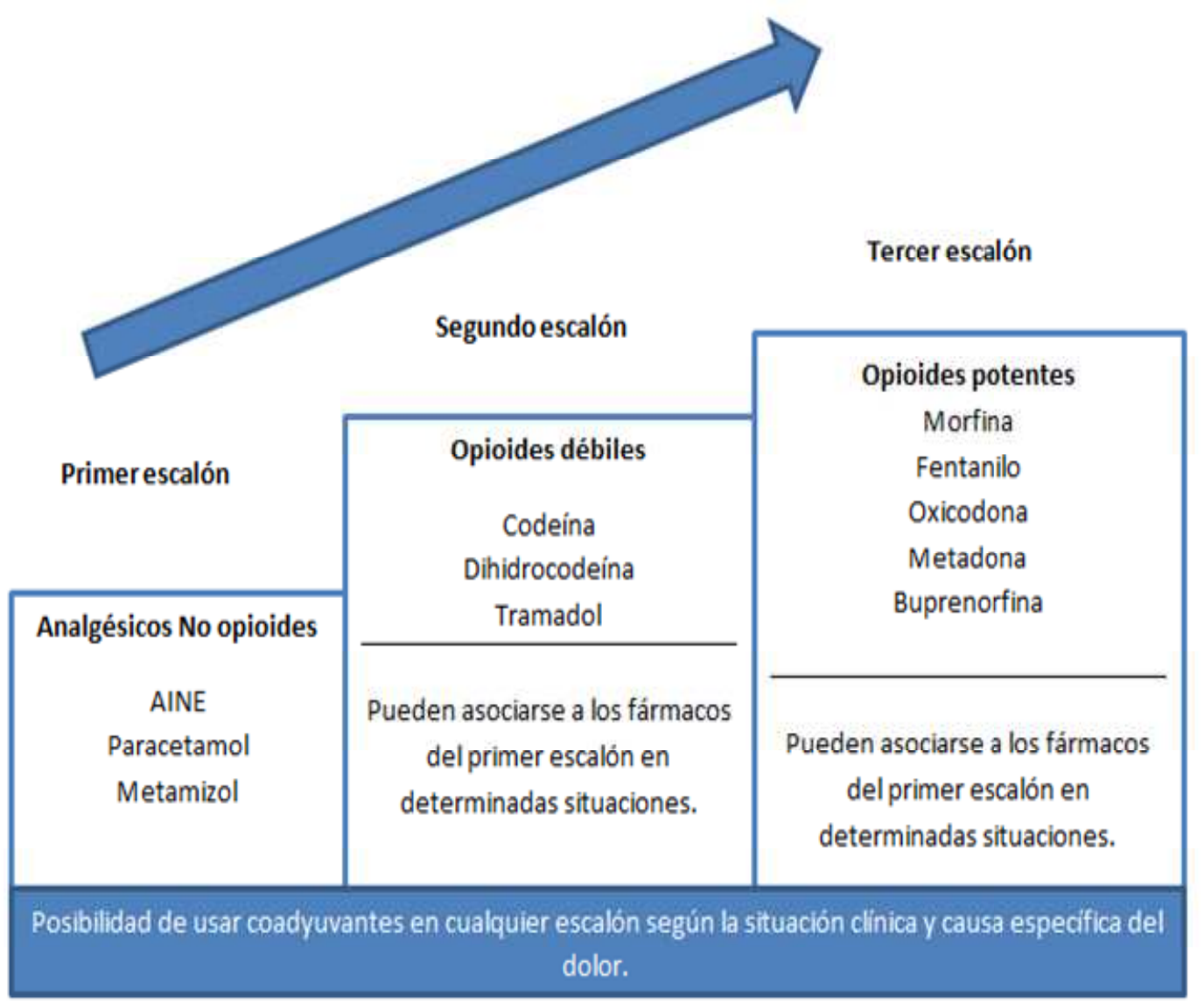

Figura No 1: Escala Analgésica de la OMS modificada. Adaptada de: World Health Organization (WHO).

Cáncer. Disponible en: http://www.who.int/cancer/palliative/painladder/en/ 
A pesar de estas guías, los numerosos avances en el campo de la medicina para el manejo del dolor han sido insuficientes; por cuanto es idea común que el dolor es solo un simple síntoma y no es perjudicial por sí mismo, además de la falta de entrenamiento en la farmacocinética de los analgésicos, el temor al potencial aditivo de efectos adversos, la dificultad de los pacientes para comunicar sus necesidades y la mala interpretación de las indicaciones médicas, lo cual hace que el manejo sea complicado, particularmente durante el período postoperatorio. Con el paso de los años, se ha encontrado evidencia científica que avala el hecho que un adecuado manejo del dolor mejora de forma considerable la satisfacción de los pacientes durante su estadía hospitalaria y contribuye a disminuir la morbi-mortalidad post quirúrgica $[5,9]$.

Por lo tanto, tratando de mejorar el control del dolor la Sociedad Americana del Dolor recomienda reconocer y tratar el dolor de manera temprana, establecer un compromiso en la evaluación del dolor, flexibilizar las dosis y combinaciones, ajustar la estrategia individualmente y educar a los profesionales a cargo $[3,10]$.

En Costa Rica son pocos los estudios realizados que analicen el abordaje del dolor en el postoperatorio, específicamente dos [11,12]. En uno de ellos, realizado en el Hospital San Juan de Dios, se analizó la concordancia en la prescripciónadministración de analgésicos durante el período postoperatorio inmediato a 60 pacientes sometidos a una cirugía ortopédica; en este se determinó la utilización más frecuente del diclofenaco y se llegó a la conclusión que la prescripción de la terapia analgésica PRN equivalente a un esquema intermitente dosisrespuesta es ineficaz. También se comprobó que la vía de administración epidural analgésica brinda un mejor control del dolor que la vía intramuscular, independientemente del tipo de cirugía evaluada [11].
El Hospital Clínica Bíblica se especializa en diversos tipos de cirugías, las cuales se clasifican en: cabeza y cuello, torácicas cardíacas y no cardíacas, abdominal superior e inferior, de espalda y de extremidades. En todas ellas es necesario fomentar un uso correcto de los analgésicos en los períodos pre, peri y post operatorio por las razones anteriormente mencionadas y por la relación directa con la correcta evolución del paciente. Por tanto, realizar un análisis comparativo de la efectividad de las estrategias terapéuticas utilizadas facilitará el establecimiento de lineamientos internos que busquen mejorar el control del dolor y la calidad en la atención, lo cual constituye la justificación primordial de este estudio. Así, el presente estudio buscó comparar la efectividad de las estrategias analgésicas de mayor uso en el manejo del dolor postoperatorio en el Hospital Clínica Bíblica durante el año 2013, tomando como hipótesis que las diferentes estrategias farmacoterapéuticas utilizadas son igualmente efectivas.

\section{MÉTODOS Y MATERIALES}

El presente estudio se basa en que existen diferentes estrategias analgésicas que buscan controlar el dolor del paciente en el postoperatorio y que los efectos combinados o aditivos de dos o más fármacos analgésicos pueden ser mayores a los obtenidos por la monoterapia. Por tanto, el objetivo principal era determinar ¿Cuáles fueron las estrategias analgésicas más efectivas en el manejo del dolor postoperatorio en el Hospital Clínica Bíblica?

Este objetivo permite establecer relación entre las siguientes variables:

a. La efectividad de las estrategias farmacoterapéuticas más utilizadas en el hospital para el manejo del dolor postoperatorio.

\section{b. Características de los pacientes y procedimientos quirúrgicos en las que las estrategias fueron utilizadas.}


Con el fin de responder a ello, se desarrolló un estudio observacional de tipo retrospectivo en que se seleccionaron a todos aquellos pacientes internados de enero a diciembre de 2013 a los cuales se administró terapia analgésica a fin de tratar el dolor postoperatorio durante su permanencia en el centro médico. Se tomó en cuenta todos aquellos pacientes mayores de 18 años con al menos 24 horas de internamiento, excluyendo a los pacientes sometidos a cirugías ambulatorias, aquellos que no reportaban EVAs en recuperación, ni el manejo del dolor transoperatorio. La información concerniente a la población en estudio se extrajo mediante consultas al Sistema Integrado de Gestión Hospitalaria (SIGH), Gestión de Paciente Hospitalizado (GPH) y/o el expediente físico del paciente.

Para cada caso particular se tomó en cuenta el tipo de procedimiento quirúrgico, edad del paciente, género, antecedentes patológicos relevantes, peso, días totales de hospitalización, tratamiento analgésico elegido en el preoperatorio, transoperatorio, tipo de anestesia, y manejo del dolor postoperatorio; además de valorar dosis, tipo de medicamentos utilizados y las combinaciones seleccionadas. Se recolectaron los EVAS del postoperatorio, detallando su fecha y hora. Se utilizó una hoja de cotejo, la cuál fue el instrumento de trabajo para la recopilación de datos.

Se verificó si la combinación de analgésicos/ antiinflamatorios fue segura, definida como la que logra aumentar la acción terapéutica buscada sin incrementar los efectos adversos derivados de mecanismos de acción similares, tomando en cuenta si la dosis era la correcta.

Estas estrategias se agruparon según tipo de cirugía y se clasificaron en 7 subtipos: cabeza y cuello, torácica no cardiaca, torácica cardiaca, extremidades, espalda/espinal, abdominal superior y abdominal inferior/ pelvis, de acuerdo con lo indicado por la guía Clinical Practice Guideline for the Management of post-operative Revista electrónica publicada por el Departamento de Farm Costa Rica, 2060 San José, Costa Rica. ${ }^{\circledR}$ All rights reserved pain y Practice Guidelines for Acute Pain Management in the Perioperative Setting, en la cual se establecen pautas sobre el abordaje más adecuado a utilizar según la clasificación y tipo de intervención quirúrgica $[3,10]$.

Para el manejo de las variables y análisis de los datos obtenidos se utilizó el programa SSPS® en su última versión. Una vez recopilada la información se determinó cuáles son las estrategias analgésicas más utilizadas en el post operatorio según procedimiento quirúrgico, y se comparó la efectividad de las mismas en términos de los EVAS obtenidos en este período. Debido al comportamiento paramétrico de las variables aplicó una prueba T-student para comparaciones entre dos grupos, y ANOVA para tres o más grupos.

Para la elaboración del presente estudio se contó con el consentimiento de la Dirección de Investigaciones Clínicas del Hospital Clínica Bíblica. Se garantiza el manejo adecuado ético y la confidencialidad de los datos de los pacientes incluidos en el estudio.

\section{PRESENTACIÓN DE RESULTADOS}

Se analizaron 701 procedimientos quirúrgicos de miembros inferiores/pelvis, los cuales fueron realizados en las salas de cirugía del Hospital Clínica Bíblica durante el período de enerodiciembre del 2013. De la población inicial $(n=789)$ se excluyeron 88 cirugías, según los criterios previamente establecidos.

En cuanto a resultados de características del paciente y estadía, el número de días de internamiento promedio de los pacientes fue de dos días (rango: 1-19 días). La edad promedio fue de 50 años (DE: 18 años, rango: 18-95 años), un $55 \%$ de pacientes eran de género femenino y un $45 \%$ de género masculino. Un 39\% manifestó la presencia de una o varias patologías concomitantes, entre las más comunes se encuentran: hipertensión arterial (10\% de los pacientes), dislipidemia e hipertensión arterial 
(3,6\%), hipertensión, diabetes mellitus, cáncer y dislipidemia (2,5\%). Un $61 \%$ de los pacientes no reporto patologías concomitantes. De los pacientes sometidos a procedimientos solo un $17 \%$ usó premedicación analgésica. La dosificación fue aplicada correctamente en el $94 \%$ de los casos en el transoperatorio. Las combinaciones más usadas en este período se muestran en la figura 2.

\section{Figura No 2: Combinaciones analgésicas más usadas en el transoperatorio.}

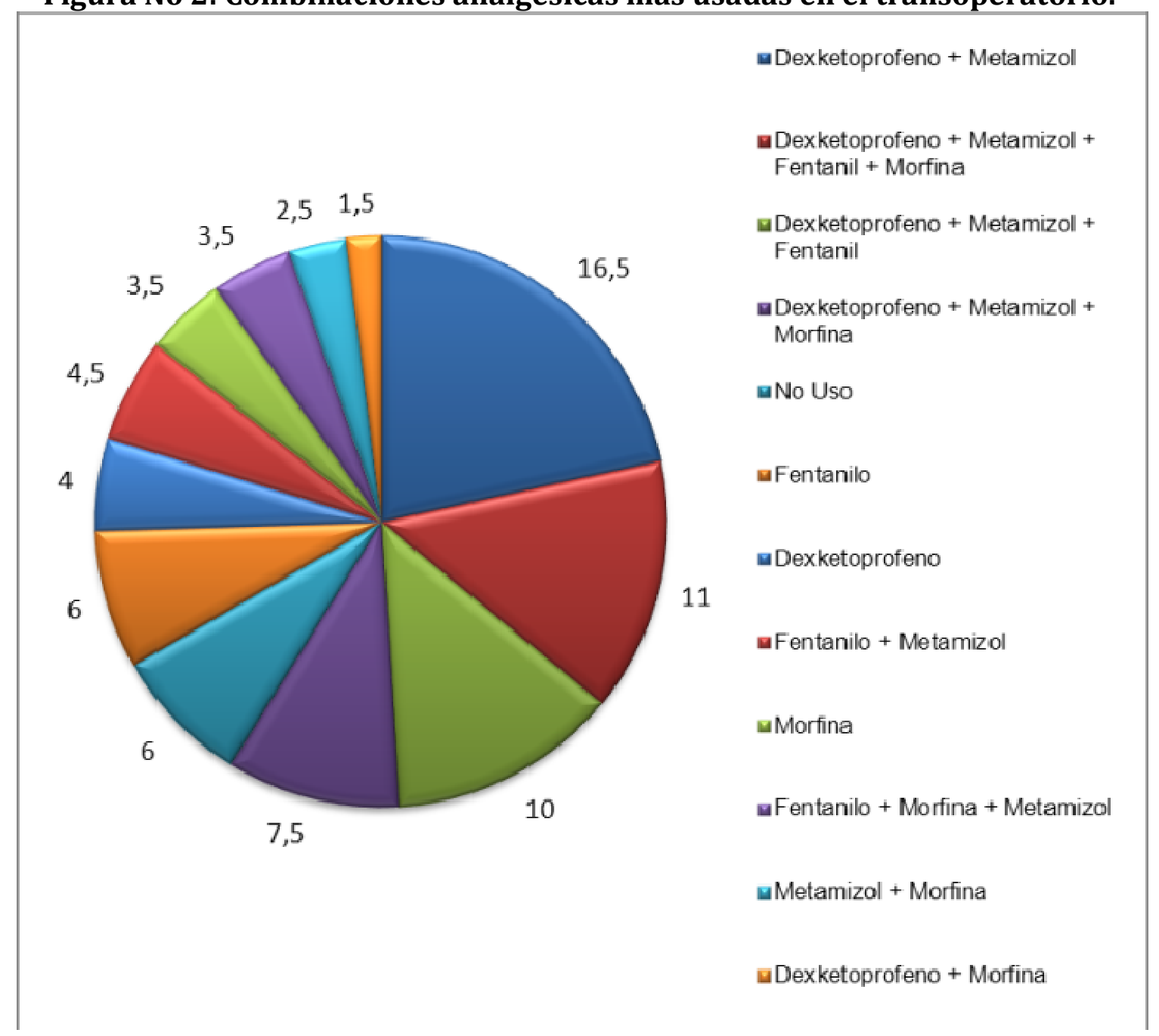

Fuente: elaboración propia.

En menos de un 5\% de las ocasiones se utilizaron combinaciones de medicamentos (analgésicos antiinflamatorios) no recomendadas o las cuales puede ser consideradas como inseguras o peligrosas.
Para el período postoperatorio las combinaciones usadas en un porcentaje superior al $2 \%$ se muestran en la figura 3. La combinación de analgésicos / antiinflamatorios utilizadas fue considerada como una combinación segura en el 
97\% de los casos. La dosis correcta fue utilizada en el $99 \%$ de los casos.

Figura No 3: Combinaciones analgésicas más usadas en el postoperatorio.

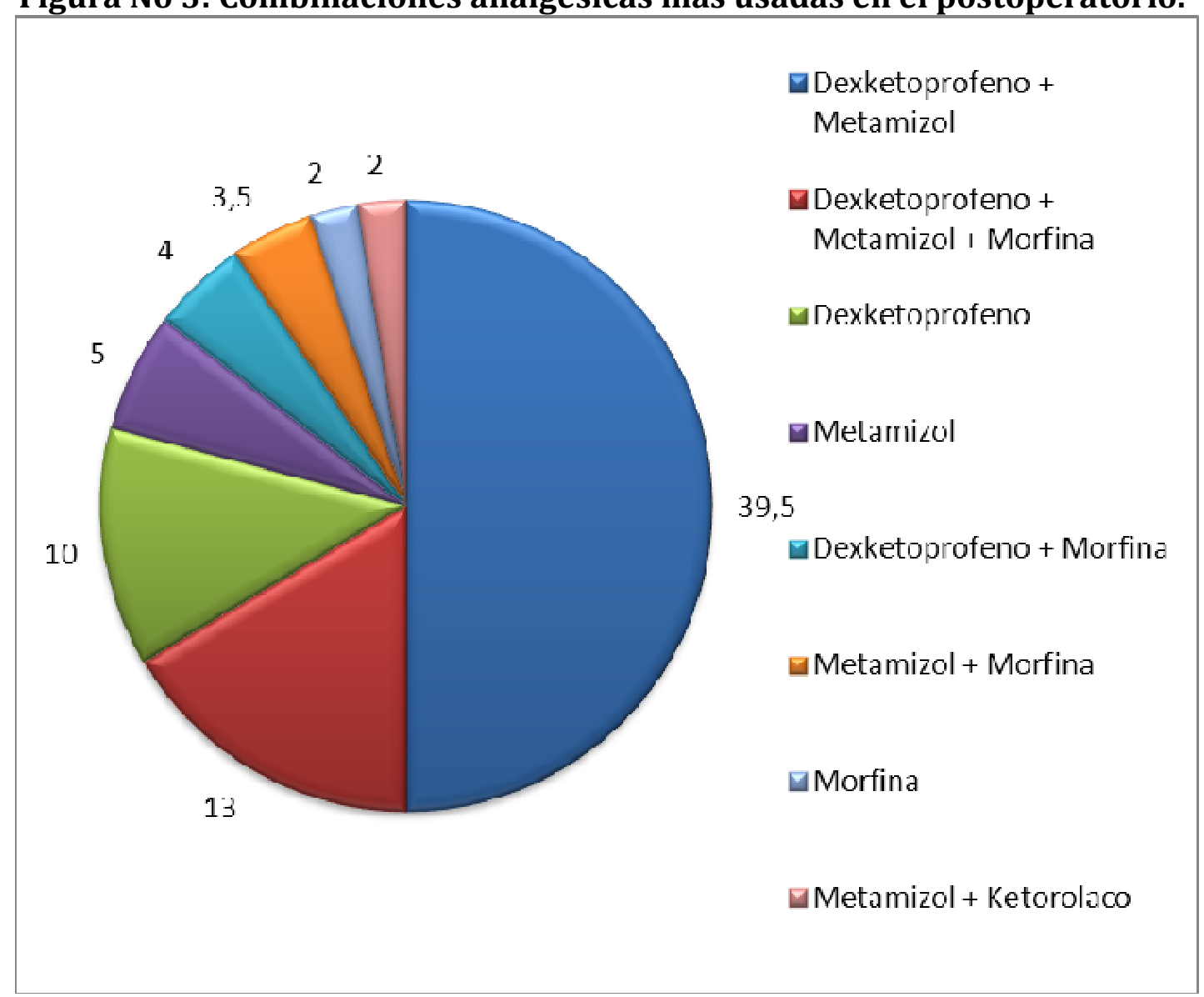

Fuente: elaboración propia.

Con respecto a la percepción del dolor durante el período de recuperación y 24 horas posteriores al procedimiento quirúrgico, el rango de valor reportado mediante el EVA, así como otros detalles al respecto se reportan en la Tabla 1.

Al comparar las tres estrategias más utilizadas en el postoperatorio (Dexketoprofeno + Metamizol (1), Dexketoprofeno + Metamizol + Morfina (2), Dexketoprofeno solo (3)) se halló que existen diferencias estadísticamente significativas entre las estrategias 1 y 2 p $=0.00008$ a favor de la combinación 1 (Dexketoprofeno + Metamizol) y la combinación 2 y 3 p=0.029 a favor de la combinación 3 (Dexketoprofeno solo) en relación con el EVA promedio de 24 horas reportado. Así mismo, no existen diferencias estadísticamente significativas entre las combinaciones 1 y 3 $\mathrm{p}=0.735$ con respecto al EVA promedio en 24 horas.

Para el período de recuperación no existen diferencias estadísticamente significativas entre ninguno de los grupos con respecto a los EVA promedio reportados. 
Para el caso de pacientes que usaron premedicación, se halló que existe una correlación entre la administración de esta y los EVAs reportados en los dos períodos (recuperación y 24 horas), con una p menor a 0.01 (EVA recuperación) y menor a 0.05 (EVA 24 horas).

Tabla No 1: Rangos de valores de EVA reportados por los pacientes durante el período de recuperación y 24 horas posterior al procedimiento quirúrgico.

\begin{tabular}{|c|c|c|}
\hline EVA & Rango & $\begin{array}{c}\text { Otros detalles } \\
\end{array}$ \\
\hline \multirow{2}{*}{$\begin{array}{c}\text { Máxima } \\
\text { Recuperación }\end{array}$} & \multirow{2}{*}{$0-10$} & $85 \%$ de los pacientes reportó valores entre $0-5$ \\
\hline & & $3,5 \%$ de los pacientes reportó valores entre $9-10$ \\
\hline $\begin{array}{c}\text { Minima } \\
\text { Recupcración }\end{array}$ & $0-6$ & 99\% de los pacientes reportó un valor de 0 \\
\hline \multirow{3}{*}{$\begin{array}{l}\text { Promedio } \\
\text { Recuperación }\end{array}$} & \multirow{3}{*}{$0-6$} & $75 \%$ de los pacientes reportó un promedio inferior a 1 \\
\hline & & $97 \%$ de los pacientes reportó valores entre 0-3 \\
\hline & & $2 \%$ de los pacientes reportó valores entre $3-6$ \\
\hline \multirow{3}{*}{$\begin{array}{c}\text { Máxima } 24 \\
\text { horas }\end{array}$} & \multirow{3}{*}{$0-10$} & $70 \%$ de los pacientes reportó un valor de 0 \\
\hline & & $84 \%$ de los pacientes reportó un valor entre 0-4 \\
\hline & & $15,5 \%$ de los pacic ntes reportó un valore cntre $5-10$ \\
\hline \multirow{3}{*}{$\begin{array}{l}\text { Minima } 24 \\
\text { horas }\end{array}$} & \multirow{3}{*}{$0-8$} & $90 \%$ dc los pacientes reportó un valor de 0 \\
\hline & & $8,5 \%$ de los pacientes reportó un valor entre $1-4$ \\
\hline & & $1 \%$ de los pacientes reportó un valor entre $5-8$ \\
\hline \multirow{3}{*}{$\begin{array}{c}\text { Promedio } 24 \\
\text { horas }\end{array}$} & \multirow{3}{*}{$0-9$} & $70 \%$ de los pacientes reportó un valor de cero \\
\hline & & $18 \%$ de los pacientes reportó un valor entre $0-3$ \\
\hline & & $8 \%$ de los pacicntes reportó un valor entre $4-9$ \\
\hline
\end{tabular}

Fuente: elaboración propia.

También se hicieron correlaciones entre los EVA para 24 horas y de recuperación en relación con variables demográficas y de hospitalización. En el caso del género, los pacientes de género masculino reportaron EVAs promedio menores que las mujeres para ambas condiciones, dicha correlación se obtuvo mediante un análisis de correlación biserial, debido a las características dicotómicas de una de las variables.

Respecto al número de días de internamiento, se halló correlación entre los EVA promedio 
obtenidos en 24 horas y los días de internamiento. Para EVAs más altos se obtuvieron mayor cantidad de días de internamiento. Por último, en relación con la edad, existe solo una correlación con los EVA promedio obtenidos, esto por cuanto entre mayor era la edad del paciente más bajos eran los EVAs reportados. El EVA en recuperación explica en un 7.9\% su varianza con respecto a la edad ( $\mathrm{p}$ inferior a 0.01). Dicho análisis fue realizado mediante una correlación de Pearson.

\section{DISCUSIÓN}

Los estudios que han analizado el manejo del dolor postoperatorio utilizando distintas combinaciones analgésicas han comprobado que existen muchos factores que influyen en el adecuado control del dolor, como lo son el tipo de procedimiento quirúrgico, la duración del procedimiento, la combinación de analgésicos utilizados antes, durante y posterior al procedimiento, la edad y la ansiedad o estado anímico del paciente, entre otros. Por tanto, la búsqueda de una combinación ideal y/o de un efecto sinérgico entre los fármacos seleccionados puede facilitar el éxito en cada una de las situaciones clínicas [1,13].

Como lo indican Mathiesen y colaboradores, existen retos en el manejo del dolor postoperatorio, no sólo en Dinamarca, sino a nivel mundial, tal como se ha observado en diversos estudios multicéntricos. Los autores proponen la necesidad de establecer estrategias de manejo de dolor específicas para cada procedimiento quirúrgico, las cuales sean el resultado de un proceso colaborativo interdisciplinario [14].

A pesar de la no existencia de un protocolo clínico que oriente a los tratantes con respecto a los medicamentos y/o combinaciones aceptables para el control del dolor en el Hospital Clínica Bíblica, tanto en aspectos de efectividad como de seguridad, los porcentajes de combinaciones inseguras hallados tanto en el periodo transoperatorio como postoperatorio se asumen como bajos ( 5 y $1 \%$, respectivamente); de hecho los valores para eficacia son menores a otros porcentajes reportados en estudios que usaron la EVA para valorar el dolor postoperatorio en cirugías de miembros, como el estudio de artroplastía de rodilla y cadera de Wylde y colaboradores, donde alrededor de un 50\% o más de los pacientes reportaron dolor de moderado a severo [15]; no obstante, es importante recalcar que deberían existir protocolos establecidos respecto a las combinaciones usadas, tal como lo plantean algunos autores [9], pues el número de estas es muy alta o, en caso contrario, establecer un sistema de monitorización de combinaciones, que evalúe si estas son adecuadas y si se están administrando en la dosificación correcta. Se debe de tomar en cuenta también que un $94 \%$ de los pacientes recibieron medicamentos en el periodo transoperatorio y esto podría haber alterado los resultados encontrados con respecto al efecto terapéutico de las combinaciones analgésicas, lo cual no fue valorado en este estudio.

Tal como se mencionó previamente, la escala visual análoga es un instrumento de valor para evaluar el manejo de dolor en un paciente, según el autoreporte de este, pues ha demostrado ser confiable, generalizable, sensible y con consistencia interna [16]. De hecho, se considera la evaluación periódica del dolor como una herramienta básica, esencial para mejorar y ajustar la farmacoterapia, además de ser de especial ayuda durante la movilización, pues valores altos podrían prolongar la convalescencia $[9,14,15]$ o incluso provocar el desarrollo de dolor crónico $[4,9,15]$. Tal es así, que hay estudios realizados para comparar esta escala con escalas numéricas y verbales. Los resultados obtenidos indican que la EVA se corresponde con valores obtenidos por escalas numéricas en la gran mayoría de los casos, siendo las excepciones calificaciones un poco más altas para la EVA en algunos estudios, así como sus limitaciones en adultos mayores o aquellos con discapacidades físicas o cognitivas [17]; no obstante, se considera más confiable que escalas verbales, las cuales presentan problemas en la comprensión de los descriptores entre idiomas $[18,19]$.

Revista electrónica publicada por el Departamento de Farmacología de la Escuela de Medicina de la Universidad de Costa Rica, 2060 San José, Costa Rica. ${ }^{\circledR}$ All rights reserved. Licensed under a Creative Commons Unported License. 
En el presente estudio, los valores promedio y mínimos se encontraron en rangos menores a un valor de 5 y 6 , que constituyen valores intermedios dentro de dicha escala. Podría asumirse, de estos datos, que el manejo es adecuado, no obstante, siempre es importante usar una batería de instrumentos y no apoyarse en uno sólo; también se debe recordar que la percepción del dolor tiene una correlación especial con la idiosincrasia del paciente, y la medición en un momento en particular no reporta el manejo del dolor durante todo el internamiento del paciente, así como tampoco sirve para comparar entre poblaciones de pacientes en un momento dado, debido a la subjetividad relacionada con la percepción del dolor [20].

La combinación más utilizada en el transoperatorio y el postoperatorio (Dexketoprofeno + Metamizol) ha demostrado en diversos estudios eficacia $y$ seguridad, lo cual apoya la selección realizada por los tratantes de los pacientes incluidos dentro del presente estudio [1,21], ya que se prefieren las estrategias multimodales que reducen no sólo el dolor durante la movilización sino también la reducción en el consumo de analgésicos opioides y las complicaciones derivadas de su uso [14].

Con respecto a las estrategias más utilizadas en el postoperatorio de 24 horas lo más importante fue que no existe una diferencia estadísticamente significativa en agregar un opiáceo a la combinación para el control del dolor o un analgésico puro como el Metamizol (para este tipo de cirugías), esto probablemente esté relacionado con el tipo de dolor generado por el procedimiento o por la eficacia de la combinación de los fármacos comparada con la adición de un opiáceo o un analgésico en estas situaciones en particular. La adición de otro fármaco en el postoperatorio en estos casos solo podría incrementar la posibilidad de manifestar efectos secundarios, tal como se ha observado o indicado en otros estudios [13]. Es por ello que se recalca la importancia de tener protocolos de manejo de dolor estandarizados según el tipo de cirugía, pues se ha demostrado en diversos estudios que las combinaciones Revista electrónica publicada por el Departamento de Farmacología de la Escuela de Medicina de la Universidad de Costa Rica, 2060 San José, Costa Rica. ${ }^{\circledR}$ All rights reserved. Licensed under a Creative Commons Unported License. analgésicas suelen ser percibidas como efectivas por los pacientes en función del dolor derivado del tipo de cirugía; así, Breivik y colaboradores no hallaron diferencias estadísticamente significativas entre acetaminofén y acetaminofén con codeína en pacientes sometidas a cesárea que tuvieran dolor moderado de línea base (4-6 en EVA) en relación con aquellas que reportaron valores de EVA superiores a 6 [19].

Se presenta una tendencia al desuso de medicamentos en el preoperatorio a nivel mundial, puesto que se ha observado la no necesidad de la utilización de los mismos en este período en específico para este tipo de procedimiento [21-23]. En el presente estudio se halló una correlación entre el uso de premedicación y valores de EVA menores; sin embargo, se debe recalcar que esta correlación sólo explica una relación proporcional en el aumento de la varianza de una variable en relación con otra y no es sinónimo de causalidad, por lo cual se requeriría la realización de estudios con una muestra mayor de pacientes que recibieron premedicación (sólo un $17 \%$ en este estudio) que analicen tanto correlación como causalidad en estas dos variables para comprobar la tendencia mundial mencionada previamente.

Similar analogía aplica para el resto de correlaciones analizadas. En el caso del género, tanto para las 24 horas posteriores como para el periodo de recuperación los EVA promedio reportado por los pacientes de género masculino fueron en promedio menores que los reportados por las mujeres sometidas a este tipo de procedimiento, aspecto que va de la mano con lo reportado por otros autores [19,23], quienes indican que las mujeres no sólo experimentan más dolor postoperatorio, sino también mayor malestar, fatiga y vómitos, además, tienen una mayor prevalencia de dolor musculoesquelético, visceral, por enfermedades autoinmunes y luego de cirugías abdominales, ortopédicas y cardiacas [24].

En el caso de la edad conforme esta aumenta se minimizan los EVAs reportados y el dolor 
percibido, aspecto también observado en otros estudios [23], así como la relación hallada entre mayores valores de EVAs promedios que acompañan a un mayor número de días de internamiento, que también se ha presentado en otras investigaciones realizadas [21-23]. En el caso de menores valores de EVA en personas adultas mayores, algunos estudios sugieren más un problema de comprensión de la escala por parte de estos, debido a que las propiedades psicométricas de la escala apuntan a una población adulta joven, por lo que en adultos mayores proponen que es más recomendable usar escalas numéricas o de descriptores verbales [4,25].

Tal vez uno de los aspectos más importantes a considerar para futuras investigaciones en el tema es no sólo valorar la severidad del dolor en términos de su intensidad, sino también en función de cualidades sensoriales del mismo y la evolución de estas antes y después de la cirugía, tal como proponen algunos autores $[15,16]$.

\section{CONCLUSIONES}

Las estrategias para el manejo del dolor postoperatorio en el Hospital Clínica Bíblica son similares a las reportadas en otros estudios, y estas fueron efectivas a pesar de la no existencia de un protocolo para el manejo del dolor en el paciente sometido a un procedimiento quirúrgico.

La efectividad de las tres estrategias más utilizadas no fue la misma, ya que idealmente se recomienda la utilización de un AINE como monoterapia para este tipo procedimientos, pero se observó que combinaciones de dos analgésicos y la ausencia de combinaciones con opioides provocaron una menor percepción de dolor en los pacientes en los dos períodos analizados.

De los resultados obtenidos se extrae que una menor percepción del dolor está relacionada con una estancia hospitalaria inferior, lo cual exige a las autoridades hospitalarias mantener un estricto control con respecto a los resultados de la terapia y la percepción del dolor de los pacientes sometidos a distintos procedimientos quirúrgicos en el tiempo.

Por último, en este estudio se halló que los hombres y los adultos mayores (indiferentemente del género) tienden a tener una menor percepción del dolor con respecto a las mujeres y adultos jóvenes.

\section{BIBLIOGRAFíA}

1. Finke DM, Schlegel HR. Dolor postoperatorio. Conceptos básicos y fundamentos para un tratamiento adecuado. Revista del Hospital General de Agudos J.M. Ramos Mejía [Internet]. 2003, 1: [19 páginas]. Disponible a través de: http://www.sld.cu/galerias/pdf/sitios/anestesiologi a/dolor_postop.pdf

2. Dolin SJ, Cashman JN, Bland JM. Effectiveness of acute postoperative pain management: I. Evidence from published data. Br J Anaesth (Internet), 2002 Sep., 89(3): (15 páginas). Disponible a través de: http://bja.oxfordjournals.org/content/89/3/409.lon g

3. The Office of Performance and Quality, VHA, Washington, DC Quality Management Directorate, United States Army MEDCOM. Clinical Practice Guideline of the management of post-operative pain. 2002.

4. Gagliese L, Katz J. Age differences in postoperative pain are scale dependent: a comparison of measures of pain intensity and quality in younger and older surgical patients. Pain. 2003; 103(1-2):11-20.

5. Li, J. Pain Management in the hospitalized patient. Med Clin North Am, 2008 Mar, 92(2): 371-385.

6. Wong DL, Hockenberry-Eaton M, Wilson D, Winkelstein ML, Schwartz, P. Wong's Essentials of Pediatric Nursing. 6a edición. St. Louis: Mosby; 2001.

7. DeLoach LJ, Higgins MS, Caplan AB, Stiff JL. The visual analog scale in the inmmediate postoperative period: intrasubject variability and correlation with a numeric scale. Anesth Analg. 1998, 86: 102-6.

8. Benzon H, Rathmell JP, Wu CJ, Turk DC, Argoff CE, Hurley RH. Practical Management of Pain. 5a edición. Elsevier; 2013.

Revista electrónica publicada por el Departamento de Farmacología de la Escuela de Medicina de la Universidad de Costa Rica, 2060 San José, Costa Rica. ${ }^{\circledR}$ All rights reserved. Licensed under a Creative Commons Unported License. 
9. Joshi GP, Schug SA, Kehlet H. Procedure-specific pain management and outcome strategies. Best Pract Res Clin Anaesthesiol. 2014, 28: 191-201.

10. The American Society of Anesthesiologists. Practice guidelines for acute pain management in the perioperative setting. Anesthesiology, 2012 Feb, 116(2):248-73.

11. Sáenz-Campos D, Sánchez-Arredondo, CM. Análisis de la concordancia prescripción-administración de analgésicos durante el postoperatorio inmediato. Acta Médica Costarricense. 2005 Octubre; 47(4): 186-191.

12. Política para el manejo de dolor. Hospital Clínica Bíblica. Comité Atención al Paciente. 2006.

13. Taylor A, Stanbury L. A review of postoperative pain management and the challenges. Current Anesthesia and Critical Care. 2009, 20 (4): 188-194.

14. Mathiesen O, Thomsen AB, Kitter B, Dahl JB, Kehlet H. Need for improved treatment of postoperative pain. Dan Med. 2012 Ap 59 (4):1-4.

15. Wylde V, Rooker J, Halliday L, Blom A. Acute postoperative pain at rest after hip and knee arthroplasty: severity, sensory qualities and impact on sleep. Orthop Traumatol Surg Res. 2011, 97:139144.

16. Galloway S, Chimhanda M, Sloan J, Anderson C, Sinacore J, Brubaker L. Pain scores are not predictive of pain medication utilization. Pain Res Treat. 2011, Article ID 987469, 5 páginas.

17. Hjermstad MJ, Fayers PM, Haugen DF, Caraceni A, Hanks GW, Loge JH, et al. Studies comparing numerical rating scales, verbal rating scales, and visual analogue scales for assessment of pain intensity in adults: a systematic literature review. J Pain Sym Man. 2010 Junio, 6 (41): 1073-1093.

18. Aicher B, Peil H, Diener H-C. Pain measurement: visual analogue scale (VAS) and verbal rating scale (VRS) in clinical trials with OTC analgesics in headache. Cephalalgia. 2011, 32(3): 185-197.

19. Breivik H, Borchgrevink PC, Allen SM, Rosseland LA, Romundstad L, Breivik Hals EK, et al. BJA. 2008, 101(1(: 17-24.

20. Wewers ME, Lowe NK. A critical review of visual analogue scales in the measurement of clinical phenomena. Research in Nursing and Health. 1990, 13, 227-236.

21. Sánchez- Carpena J, Dominguez- Hervella F, García I, Gene E, Bugarín R, Marín A, et al. Comparison of intravenous dexketoprofen and dipyrone in acute renal colic. Eur J Clin Pharmacol. Epub 2007 Jun, 63(8): 751-760.

22. Ong CK, Lirk P, Seymour RA, Jenkins BJ. The efficacy of preemptive analgesia for acute postoperative pain management: A metaanalysis. Anesth Analg. 2005 Mar, 100(3): 757-73.

23. Alabas OA, Tashani OA, Tabasam G, Johnson MI. Gender role affects experimental pain responses: A systemic review with metaanalysis. Eur J Pain. 2012 Oct, 16(9): 1211-23.

24. Tolver MA, Strandfelt $\mathrm{P}$, Rosenberg J, Bisgaard T. Female gender is a risk factor for pain, discomfort, and fatigue after laparoscopic groin hernia repair. Hernia. 2013 Junio, 17 (3): 321-27.

25. Falzone E, Hoffmann C, Keita H. Postoperative analgesia in elderly patients. Drugs Aging. 2013, 30 (2): 81-90.

\section{INFORMACIÓN DEL AUTOR}

Chaverri Fernández, José M.

Email.: jose.chaverri@ucr.ac.cr 